\title{
Management of Acute Pulmonary Embolism With a Pulmonary Embolism Response Team
}

\author{
Jason R. Cuomo, MD, Vishal Arora, MD, and Thad Wilkins, MD
}

Despite recent advances in the assessment, risk stratification, and treatment of acute pulmonary embolism (PE), it remains a leading cause of cardiovascular morbidity and mortality in the United States each year. Patient presentation and prognosis are heterogeneous, and a variety of diagnostic and therapeutic instruments have arisen to assist in providing patients with the appropriate level of care and aggressiveness of approach. Fortunately, a growing number of institutions now have pulmonary embolism response teams (PERT) that urgently assist with risk assessment and management of patients with massive and sub-massive PE. In service of providers at the point of contact with acute PE, this review aims to summarize the data pertinent to rapid risk assessment and the interpretation of diagnostics used to that end. The role of PERT and the indications for systemic fibrinolysis and invasive therapies are also discussed. (J Am Board Fam Med 2021;34:402-408.)

Keywords: Cardiology, Clinical Decision-Making, Echocardiography, Fibrinolysis, Patient Care Team, Pulmonary Embolism, Pulmonary Medicine, Radiology, Risk Assessment, Thrombolytic Therapy, Venous Thromboembolism

Despite recent advances in the assessment, risk stratification, and treatment of acute pulmonary embolism (PE), it remains a leading cause of cardiovascular morbidity and mortality, resulting in over 350000 hospitalizations and over 100000 deaths in the United States each year. ${ }^{1,2}$ The indirect costs of $\mathrm{PE}$ and venous thromboembolic events are also considerable, both concerning increased rates of long-term disability and decreased quality of life. ${ }^{3}$ The distribution of these insults among patients with $\mathrm{PE}$ is heterogenous in the extreme. Treatment options include outpatient management with oral anticoagulation, administration of high-risk fibrinolytic therapy, catheterdirected thrombolysis, or cardiothoracic surgery. ${ }^{4}$ Finally, as our understanding of acute PE and its chronic sequelae has deepened, a carefully selected

This article was externally peer reviewed.

Submitted 23 June 2020; revised 13 September 2020; accepted 14 September 2020.

From the Division of Cardiology, Yale University, New Haven, CT (JC); Division of Cardiology, Medical College of Georgia at the Augusta University Medical Center (VA); and the Department of Family Medicine, Medical College of Georgia at the Augusta University Medical Center, Augusta (TW).

Funding: None.

Conflict of interest: The authors report are no conflicts of interest.

Corresponding author: Thad Wilkins, MD, MBA, FAAFP, Medical College of Georgia at Augusta University, 1120 15th Street, Augusta, GA, 30912 (E-mail: jwilkins@augusta.edu). intermediate-risk group of patients has been identified who experience morbidity and mortality benefits following targeted invasive therapies. ${ }^{5-7}$

It follows that clinical decision-making in acute $\mathrm{PE}$ is complex, and the presence of systemic fibrinolytics in the decision-tree guarantees that the stakes are high. Fortunately, PE has remained an area of active research in recent years, and a variety of tools are now available to assist providers on the front lines in accurately distinguishing between these presentations. The proliferation of pulmonary embolism response teams (PERT) at tertiary centers throughout the United States provides a rapid subspecialty response in intermediate- and high-risk cases. ${ }^{8}$ The present article aims to examine the following recommendations: (a) furnish providers at the point of contact with acute $\mathrm{PE}$ with the means to perform an accurate risk assessment, (b) review the data directing the use of systemic fibrinolysis in high-risk patients, and (c) provide an overview of the PERT process, with emphasis on how patients are selected for advanced therapies, highlighting the crucial role of providers at the point-of-contact in re-stratifying patients as "intermediate-low" and "intermediate-high" risk.

\section{Definitions}

The American Heart Association (AHA) divides patients presenting with acute PE into 3 similar 
groups according to their risk of near-term mortality. ${ }^{11}$ The majority of this risk is borne by the 5 to $10 \%$ of patients who present with sustained hypotension. ${ }^{9 \cdot 10}$ These are the patients with "massive" or "high-risk" PE, which the AHA defines as acute $\mathrm{PE}$ associated with any of the following 3 features: (1) hypotension defined as a systolic blood pressure $<90 \mathrm{mmHg}$ sustained for at least 15 minutes, (2) a drop of $>40 \mathrm{mmHg}$ in systolic blood pressure for at least 15 minutes, or (3) a vasopressor requirement. ${ }^{1{ }^{1 / 12}}$ Massive PE is associated with in-hospital and 90 -day mortality rates approaching $25 \%$ and $50 \%$, respectively, when treated with anticoagulation alone. ${ }^{9,13,14}$ Early and aggressive treatment with systemic fibrinolytics has been shown to halve the near-term mortality associated with massive $\mathrm{PE}$, which translates to an absolute reduction in mortality of over $10 \% .^{14}$

While mortality among normotensive patients with $\mathrm{PE}$ is much lower, an intermediate-risk subgroup is characterized by the presence of right heart strain or myocardial necrosis. PE associated with right ventricular (RV) injury or dysfunction is designated "submassive" and is associated with a 30-day mortality of $3 \%$ to $5 \%$ when treated with anticoagulation alone. ${ }^{15,16}$ There is little data regarding long-term sequelae specific to submassive PE. However, dyspnea and chronic thromboembolic pulmonary hypertension (CTEPH) were recently reported at 3-year follow-up in $33 \%$ and $2.7 \%$ of the PEITHO trial subjects, respectively. ${ }^{17} \mathrm{CTEPH}$ is uncommon, complicating less than $4 \%$ of cases. ${ }^{17,18}$ Outcomes data regarding the use of systemic thrombolytics in this population have been inconsistent, with 1 high-quality randomized controlled trial finding no benefit due to increased major bleeding events. ${ }^{16}$ A subsequent meta-analysis suggested evidence of benefit with careful patient selection. ${ }^{15}$ Both massive and submassive $\mathrm{PE}$ demonstrate improved hemodynamics and right ventricular function in the days after therapy, though long-term outcomes remain uncertain. ${ }^{19,20}$

Finally, the remaining $50 \%$ to $60 \%$ of patients with $\mathrm{PE}$ comprise the low-risk group, which is characterized by the absence of hemodynamic instability, RV injury, or RV dysfunction. Their 30-day mortality due to $\mathrm{PE}$ is less than $1 \%$, long-term sequelae are typically absent, and outpatient treatment with oral anticoagulants is often appropriate. ${ }^{21}$

As recently as a decade ago, 2 notable problems remained unaddressed by this scheme. First, systemic fibrinolytic therapy is associated with an absolute excess risk of major bleeding of $6 \%$ to $12 \%$ and with intracranial bleeding rates of $0.5 \%$ to $3 \% .^{14,16,22,23}$ In patients with contraindications to fibrinolysis the risks may be much higher, and yet $40 \%$ of patients in 1 high-quality study received this therapy despite their presence. ${ }^{13}$ This finding underscores both the high prevalence of risk factors for bleeding in this patient population and the desperate position in which the patient and physician often found themselves when faced with massive PE. Second, though these high rates of major bleeding make systemic fibrinolytic therapy a dangerous option for routine use in intermediate-risk patients, treatment with anticoagulation alone results in unacceptable morbidity and mortality for many of these patients.

\section{The PERT Process}

The first Pulmonary Embolism Response Team (PERT) was developed in 2012 to fill these practice gaps. Armed with a variety of surgical and catheterbased interventions as well as multimodality imaging, PERTs provide a rapid response to hemodynamically significant acute $\mathrm{PE}$, streamlining multidisciplinary consultation and resource mobilization for patients with massive or submassive $\mathrm{PE}$ in the inpatient or emergency department settings. ${ }^{24}$ PERT programs now function in over 100 hospitals in the United States, where they consist of subspecialists from interventional cardiology, interventional radiology, critical care, cardiothoracic surgery, and vascular medicine, among others. ${ }^{8}$

While subspecialty participation and PERT protocols differ somewhat between institutions, the PERT Consortium has provided standardization by way of diagnostic and treatment algorithms. ${ }^{5}$ In general, PERT activation occurs after acute PE is diagnosed by computed tomography angiography (CTA) in patients suspected to be at intermediate or high risk of near-term mortality based on clinical presentation, elevated cardiac biomarkers, or evidence of right heart strain on echocardiography or CTA. The designated responder makes a prompt and focused evaluation of the patient, with the goals of risk stratifying the patient for 30-day mortality while assessing for contraindications to first-line therapy. If interventional therapy is warranted, selection of the optimal modality is made in the multidisciplinary discussion, and recommendations are given regarding outstanding diagnostics as well 
as anticoagulation, pressor support, and the necessary level of care. ${ }^{5}$

\section{The PERT Assessment}

Acute PE threatens life by imposing an intolerable pressure-load on the right ventricle (RV). When large enough, the pulmonary embolus serves both as a mechanical outflow obstruction and a cause of microvascular vasoconstriction in the pulmonary circuit, impairing cardiac output and gas exchange, and resulting in the hypoxia and circulatory collapse that are characteristic of this state. It follows that the PERT responder's first and most important task is to generate a comprehensive assessment of the risk of catastrophic RV failure, stratifying them as having a high, intermediate, or low risk of 30-day mortality from this cause by direct and indirect assessment of RV health and function.

The most critical prognosticator is the presence or absence of sustained systemic hypotension, as it is the most emergent finding in acute PE save for sudden cardiac death and is associated with a 3 -fold increase in mortality versus normotensive, symptomatic PE. ${ }^{22,25}$ In hypotensive patients with acute PE, causality must be established before the diagnosis of massive PE is made, and alternative causes of hypotension must be excluded. Findings of RV dilation and dysfunction on echocardiography are supportive, as are the presence of saddle embolus or large thrombus burden on the diagnostic CTPA. Once the diagnosis of massive $\mathrm{PE}$ is confirmed, a rapid assessment for contraindications to systemic fibrinolysis is the last critical step before definitive therapy.

In normotensive patients, assessment of RV health often begins with a study that has already been performed, as several CTPA findings are robustly predictive of adverse short-term outcomes. These include abnormal position of the interventricular septum, reflux of contrast into the inferior vena cava, and indices of RV enlargement. In a prospective study of CTPA for risk assessment in patients with acute $\mathrm{PE}$, a ratio of $\mathrm{RV}$-to-left ventricular (LV) diameters greater than 1.0 was found to have a sensitivity of $85 \%$ for 30 -day mortality due to $\mathrm{PE}$, though specificity was poor. ${ }^{26} \mathrm{~A}$ ratio of RV-to-LV 3 Days volumes greater than 1.2 was equally sensitive for this outcome. The most specific finding, reflux of contrast into the inferior vena cava $(86 \%)$, is seldom seen in other hypotensive states and may assist the clinician in excluding alternative causes of hemodynamic compromise. In patients with a small thrombus burden on CTPA (ie, subsegmental PE), the absence of these findings is often sufficient to stratify the patient as low risk.

The PERT responder's assessment continues with clinical data gathering, and the estimation of risk by either of 2 well-validated risk scores, the Pulmonary Embolism Severity Index (PESI) and the Simplified PESI. The PESI relies on the assessment of 11 clinical variables, including age, history of cancer, and vital signs to categorize patients into 5 risk classes. ${ }^{27}$ Developed in 2005, the PESI is a highly sensitive instrument for prediction of 30-day mortality. ${ }^{27,28}$ The Simplified PESI was derived by subjecting each of the clinical criteria to a univariate logistic regression and removing those that did not correlate significantly with death at 30 days. ${ }^{29}$ When scores corresponding to risk classes I and II are considered as negative results (ie, the "reference range"), both indices boast sensitivities exceeding 90\% and negative predictive values exceeding $98 \%$. Taken in conjunction with negative echocardiographic, serum, and CT indices of RV injury, a low-risk PESI or Simplified PESI score furnishes a compelling argument for medical therapy (Table 1).

Like the PESI and sPESI, biomarker data function in the PERT assessment as high-sensitivity, low-specificity predictors of PE-mediated morbidity and mortality. The best-studied serum biomarker is the high-sensitivity troponin- $T$ assay, which was found earlier in the decade to predict 30day mortality with $87 \%$ sensitivity when a cutoff of $14 \mathrm{pg} / \mathrm{mL}$ is used. ${ }^{30}$ More recently, another prospective study confirmed this finding while also suggesting a cutoff of $45 \mathrm{pg} / \mathrm{mL}$ for patients older than 75 years to enhance specificity. ${ }^{31} \mathrm{~N}$-terminal pro-brain natriuretic peptide has a similar sensitivity for the prediction of 30-day mortality and adverse events. ${ }^{32}$

Finally, the echocardiogram is crucial to the assessment of patients with $\mathrm{PE}$ and is often performed urgently following PERT activation. Echocardiography is sometimes used to assist in the diagnosis of $\mathrm{PE}$, as up to $20 \%$ of PERT activations occur in the context of a high-risk patient for whom a CT diagnosis is unavailable, that is, due to renal insufficiency or contrast allergy. ${ }^{33}$ In other cases, it is used to confirm that significant symptoms or 
Table 1. Original and Simplified Pulmonary Embolism Severity Indices

\begin{tabular}{|c|c|c|}
\hline Parameter & PESI & Simplified PESI \\
\hline Age & Age in years & 1 point if age $>80$ years \\
\hline Male sex & +10 points & - \\
\hline Cancer & +30 points & 1 point \\
\hline Chronic heart failure & +10 points & 1 point \\
\hline Chronic pulmonary disease & +10 points & \\
\hline Pulse rate $\geq 110 \mathrm{BPM}$ & +10 points & 1 point \\
\hline Systolic blood pressure $<100 \mathrm{~mm} \mathrm{Hg}$ & +30 points & 1 point \\
\hline Respiratory rate $>30$ breaths per minute & +20 points & - \\
\hline Temperature $<36^{\circ} \mathrm{C}$ & +20 points & - \\
\hline Altered mental status & +60 points & - \\
\hline \multirow[t]{2}{*}{ Arterial oxyhemoglobin saturation $90 \%$} & +60 points & 1 point \\
\hline & \multicolumn{2}{|c|}{ Estimated 30-day Mortality } \\
\hline Adapted from Konstantinides SV, Meyer G, et al, 2019. & $\begin{array}{l}\text { Class I, } \leq 65 \text { points: } \\
\bullet \text { Very low risk: } 0-1.6 \% \\
\text { Class II, } 66-85 \text { points: } \\
\text { - Low risk: } 1.7-3.5 \% \\
\text { Class III, } 86-105 \text { points } \\
\text { - Moderate risk: } 3.2-7.1 \% \\
\text { Class IV: } 106-125 \text { points: } \\
\bullet \text { High risk: } 4.0-11.4 \% \\
\text { Class V: }>125 \text { points } \\
\text { - Very high risk: } 10.0-24.5 \%\end{array}$ & $\begin{array}{l}0 \text { points: } 1.0 \% \\
\geq 1 \text { point(s): } 10.9 \%\end{array}$ \\
\hline
\end{tabular}

PESI, Pulmonary Embolism Severity Index.

hemodynamic abnormalities, etc., are attributable to $\mathrm{PE}$ and not to some other cause. Its most common application, however, is to further risk-stratify patients with intermediate-risk PE. Two echocardiographic measurements have been validated for this purpose. As with CTA, the ratio of $\mathrm{RV}$-to- $\mathrm{LV}$ size is assessed, though it is less sensitive and specific in this setting, ${ }^{34}$ likely due to the operator-dependence of echo. The clear advantage lies with the assessment of RV systolic function, particularly through measurement of its surrogate marker which is tricuspid annular plane systolic excursion (TAPSE). In a large, prospective study of patients with low- and intermediate-risk $\mathrm{PE}$, abnormal TAPSE values $\leq 1.6 \mathrm{~cm}$ were associated with a hazard ratio of 4.4 for 30 -day mortality. ${ }^{35}$ In another prospective study of a similar patient population, TAPSE measurements $\geq 2.0 \mathrm{~cm}$ had a negative predictive value of $100 \%$ for the clinical endpoint and were sufficient to re-stratify patients as low-risk for PE-associated death and rescue thrombolysis. Conversely, a TAPSE $\leq 1.5 \mathrm{~cm}$ was associated with a positive predictive value of $23 \%$ and a hazard ratio of 27.9 for this combined endpoint. ${ }^{36}$ This measurement is easily obtained in most patients and is highly reproducible, accounting for its success in prognostication for PE.

\section{From Assessment to Treatment}

The PERT responder's risk assessment determines whether the patient is to be treated with systemic fibrinolytics, catheter-directed fibrinolytics, mechanical thrombectomy, or anticoagulation alone. A detailed account of the interventional management of acute PE would go beyond the scope of the present discussion, but a review of a few key concepts is warranted. For patients with high-risk PE and no risk factors for bleeding, the risk of mortality is high enough to warrant treatment with the maximum-efficacy agent, which is systemic fibrinolysis. However, when contraindications to systemic fibrinolysis are present, or when the treatment fails to restore hemodynamic stability, catheter-directed fibrinolysis provides the ability to administer low-dose fibrinolytics to the site of the thrombus. This treatment protocol requires an infusion of recombinant tissue plasminogen activator over 6 to 12 hours through catheters inserted into the right and/or left pulmonary arteries via the femoral or jugular veins. The total fibrinolytic dose is much lower than that utilized in systemic fibrinolysis, and major bleeding and intracranial hemorrhage occur only rarely (Table 2$)., 7$

This approach attenuates bleeding risk sufficiently in most patients, but when absolute contraindications to fibrinolytic therapy are present, as in 
Table 2. Absolute and Relative Contraindications for Administration of Thrombolytic Therapy (Systemic and Local)*

\begin{tabular}{|c|c|}
\hline Absolute Contraindications & Relative Contraindications \\
\hline $\begin{array}{l}\text { Structural intracranial disease } \\
\text { Previous intracranial hemorrhage } \\
\text { Ischemic stroke within } 3 \text { months (unless occurring in the prior } 3 \text { hours) } \\
\text { Recent head trauma with fracture or brain injury } \\
\text { Recent brain or spinal surgery } \\
\text { Active bleeding (excluding menses) } \\
\text { Bleeding diathesis }\end{array}$ & $\begin{array}{l}\text { Systolic BP > }>180 \\
\text { Diastolic BP }>110 \\
\text { Recent bleeding (non-intracranial, excluding menses) } \\
\text { Recent surgery } \\
\text { Q } \text { Recent invasive procedure } \\
\text { Chronically Anticoagulated } \\
\text { Q Traumatic cardiopulmonary resuscitation } \\
\text { Pericarditis or pericardial fluid } \\
\text { Diabetic retinopathy } \\
\text { Pregnancy } \\
\text { Age }>75 \text { y } \\
\text { Low body weight (e.g. }<60 \mathrm{~kg}) \\
\text { Female } \\
\text { Black race }\end{array}$ \\
\hline
\end{tabular}

*Adapted from Kearon C, Akl EA, Ornelas J, et al, 2016. ${ }^{39}$

a patient with a history of intracranial hemorrhage or recent stroke, catheter-directed mechanical thrombectomy (CDMT) may be preferred to fibrinolysis. Thrombectomy may also be preferred in patients for whom hemodynamic instability argues against reliance on slow-infusion catheters for hemodynamic improvement. ${ }^{37}$ CDMT is typically performed using an aspiration catheter or with a rheolytic device that fragments the thrombus with a saline jet before aspiration. Finally, when the thrombus burden and bleeding risk are both particularly high, surgical embolectomy may be considered.

The optimal management of normotensive patients with pulmonary embolism is not well- defined in the literature, and disagreement exists even among professional societies. Robust studies of catheter-directed fibrinolysis in patients with submassive $\mathrm{PE}$ and imaging evidence of $\mathrm{RV}$ enlargement have demonstrated decreases in RV size and PA pressure during the index hospitalization in excess of those seen in patients treated with therapeutic anticoagulation alone. Still, data regarding the durability of these benefits are lacking, and no mortality benefit has been reported. ${ }^{6,7}$ Nevertheless, the most recent guideline statements of the European Society of Cardiology and the PERT Consortium recommend that it be considered in select populations, particularly those with

Figure 1A. Algorithm for risk assessment and treatment of acute PE*

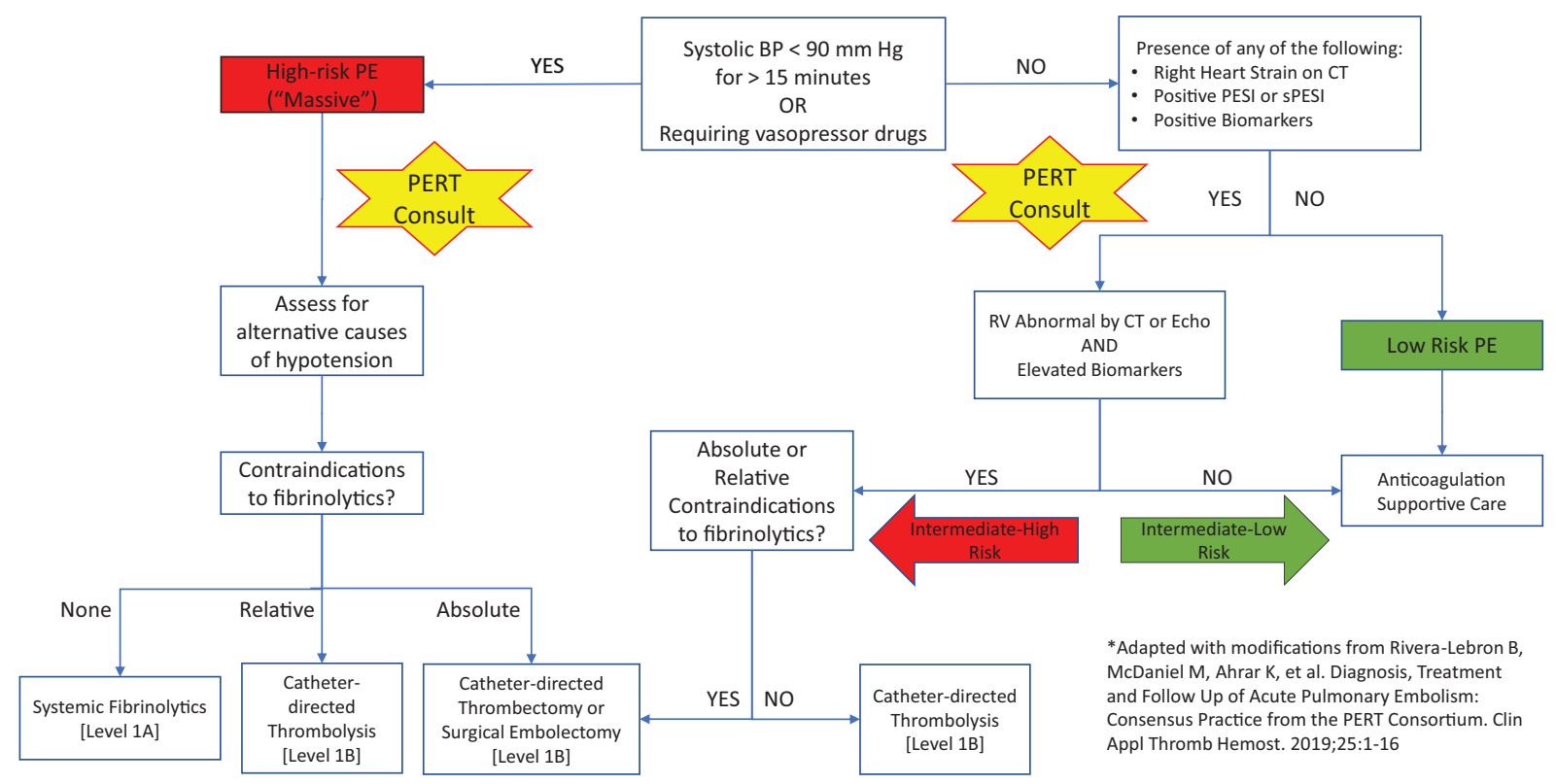


massive or submassive $\mathrm{PE}$ and contraindications to systemic fibrinolysis ${ }^{5,38}$. Our proposed algorithm (Figure 1A) follows theirs in distinguishing between "intermediate-high" and "intermediatelow" risk treatment groups within the broader category of intermediate mortality risk. Accordingly, we recommend that normotensive patients be considered for invasive therapy who have an intermediate mortality risk, positive biomarkers, and imaging evidence of RV dysfunction.

\section{Conclusion}

Systemic fibrinolysis is beneficial and potentially lifesaving for patients with massive and submassive pulmonary embolism, but it carries a significant risk of major bleeding and is often contraindicated. Catheterdirected techniques are frequently used in high-risk patients at elevated bleeding risk and in a subgroup of intermediate-risk patients in whom are found both biomarker evidence of myocardial injury and imaging evidence of right heart strain. While the burgeoning presence of PERT programs in the United States promises increased access to a rapid subspecialty response to intermediate- and high-risk PE, risk assessment begins at the first point of contact with these patients and can be potentially lifesaving.

To see this article online, please go to: http://jabfm.org/content/ 34/2/402.full.

\section{References}

1. Office of the Surgeon General (US); National Heart, Lung, and Blood Institute (US). The Surgeon General's Call to Action to Prevent Deep Vein Thrombosis and Pulmonary Embolism. Rockville (MD): Office of the Surgeon General (US); 2008. Available at: https://www.ncbi.nlm.nih. gov/books/NBK44178. Accessed March 26, 2021.

2. Centers for Disease Control and Prevention. Venous Thromboembolism in Adult Hospitalizations United States, 2007-2009. MMWR 2012;61:401-23. Available at: https://www.cdc.gov/mmwr/preview/ mmwrhtml/mm6122a1.htm. Accessed March 26, 2021.

3. Braekkan SK, Grosse SD, Okoroh EM, et al. Venous thromboembolism and subsequent permanent work-related disability. J Thromb Haemost 2016;14:1978-87.

4. Piran S, Le Gal G, Wells PS, et al. Outpatient treatment of symptomatic pulmonary embolism: a systematic review and meta-analysis. Thromb Res 2013;132:515-9.

5. Rivera-Lebron B, McDaniel M, Ahrar K, PERT Consortium, , et al. Diagnosis, Treatment and Follow
Up of Acute Pulmonary Embolism: Consensus Practice from the PERT Consortium. Clin Appl Thromb Hemost 2019;25:1076029619853037-16.

6. Kucher N, Boekstegers P, Müller OJ, et al. Randomized, controlled trial of ultrasound-assisted catheter-directed thrombolysis for acute intermediate-risk pulmonary embolism. Circulation 2014;129: 479-86.

7. Piazza G, Hohlfelder B, Goldhaber S, Piazza G, Ohlfelder B, Jaff MR, et al. A prospective, singlearm, multicenter trial of ultrasound-facilitated, catheter-directed, low-dose fibrinolysis for acute massive and submassive pulmonary embolism: the SEATTLE II study. JACC Cardiovasc Interv 2015;8:1382-92.

8. Todoran TM, Giri J, Barnes GD, PERT Consortium, et al. Treatment of submassive and massive pulmonary embolism: a clinical practice survey from the second annual meeting of the Pulmonary Embolism Response Team Consortium. J Thromb Thrombolysis 2018;46:39-49.

9. Kucher N, Rossi E, Rosa M, Goldhaber S. Massive pulmonary embolism. Circulation 2006;113:577-82.

10. Secemsky E, Chang Y, Jain CC, et al. Contemporary management and outcomes of patients with massive and submassive pulmonary embolism. Am J Med 2018;131:1506-14.e0.

11. Giri J, Sista A, Weinberg I, et al. Interventional therapies for acute pulmonary embolism: current status and principles for development of novel evidence: a scientific statement from the American Heart Association. Circulation 2019;140:e774e801.

12. Jaff MR, McMurtry MS, Archer SL, American Heart Association Council on Arteriosclerosis, Thrombosis and Vascular Biology, et al. Management of massive and submassive pulmonary embolism, iliofemoral deep vein thrombosis, and chronic thromboembolic pulmonary hypertension: a scientific statement from the American Heart Association [published correction appears in Circulation. 2012 Aug 14;126(7):e104] [published correction appears in. Circulation. 2012 Mar 20;125(11):e495]. Circulation 2011;123:1788830.

13. Kasper W, Konstantinides S, Geibel A, et al. Management strategies and determinants of outcome in acute major pulmonary embolism: results of a multicenter registry. J Am Coll Cardiol 1997;30:1165-71.

14. Wan S, Quinlan DJ, Agnelli G, Eikelboom JW. Thrombolysis compared with heparin for the initial treatment of pulmonary embolism: a meta-analysis of the randomized controlled trials. Circulation 2004;110:744-9.

15. Chatterjee S, Chakraborty A, Weinberg I, et al. Thrombolysis for pulmonary embolism and risk of all-cause mortality, major bleeding, and intracranial hemorrhage: a meta-analysis. JAMA 2014;311: 2414-21. 
16. Meyer G, Vicaut E, Danays T, et al. Fibrinolysis for patients with intermediate-risk pulmonary embolism. N Engl J Med 2014;370:1402-11.

17. Konstantinides S, Vicaut E, Danays T, et al. Impact of thrombolytic therapy on the long-term outcome of intermediate-risk pulmonary embolism. J Am Coll Cardiol 2017;69:1536-44.

18. Pengo V, Lensing AW, Prins MH, Thromboembolic Pulmonary Hypertension Study Group, et al. Incidence of chronic thromboembolic pulmonary hypertension after pulmonary embolism. N Engl J Med 2004;350:2257-64.

19. Goldhaber SZ, Haire WD, Feldstein ML, et al. Alteplase versus heparin in acute pulmonary embolism: randomised trial assessing right-ventricular function and pulmonary perfusion. Lancet 1993; 341:507-11.

20. Konstantinides S, Tiede N, Geibel A, Olschewski M, Just H, Kasper W. Comparison of alteplase versus heparin for resolution of major pulmonary embolism. Am J Cardiol 1998;82:966-70.

21. Aujesky D, Roy PM, Verschuren F, et al. Outpatient versus inpatient treatment for patients with acute pulmonary embolism: an international, open-label, randomised, non-inferiority trial. Lancet 2011;378:41-8.

22. Goldhaber SZ, Visani L, De Rosa M. Acute pulmonary embolism: clinical outcomes in the International Cooperative Pulmonary Embolism Registry (ICOPER). Lancet 1999;353: 1386-9.

23. Fiumara K, Kucher N, Fanikos J, Goldhaber SZ. Predictors of major hemorrhage following fibrinolysis for acute pulmonary embolism. Am J Cardiol 2006;97:127-9.

24. Rosovsky R, Chang Y, Rosenfield K, et al. Changes in treatment and outcomes after creation of a pulmonary embolism response team (PERT), a 10year analysis. J Thromb Thrombolysis 2019;47: 31-40.

25. Laporte S, Mismetti P, Décousus H, the RIETE Investigators, et al. Clinical predictors for fatal pulmonary embolism in 15,520 patients with venous thromboembolism: findings from the Registro Informatizado de la Enfermedad TromboEmbolica venosa (RIETE) Registry. Circulation 2008;117:1711-6.

26. Kang DK, Thilo C, Schoepf UJ, et al. CT signs of right ventricular dysfunction: prognostic role in acute pulmonary embolism. JACC Cardiovasc Imaging 2011;4:841-9.

27. Aujesky D, Obrosky DS, Stone RA, et al. Derivation and validation of a prognostic model for pulmonary embolism. Am J Respir Crit Care Med 2005;172:1041-6.
28. Aujesky D, Roy PM, Le Manach CP, et al. Validation of a model to predict adverse outcomes in patients with pulmonary embolism. Eur Heart J 2006;27:476-81.

29. Jiménez D, Aujesky D, Moores L, RIETE Investigators, et al. Simplification of the pulmonary embolism severity index for prognostication in patients with acute symptomatic pulmonary embolism. Arch Intern Med 2010;170:1383-9.

30. Lankeit $M$, Jiménez D, Kostrubiec $M$, et al. Predictive value of the high-sensitivity troponin T assay and the simplified Pulmonary Embolism Severity Index in hemodynamically stable patients with acute pulmonary embolism: a prospective validation study. Circulation 2011;124:2716-24.

31. Kaeberich A, Seeber V, Jiménez D, et al. Ageadjusted high-sensitivity troponin $\mathrm{T}$ cut-off value for risk stratification of pulmonary embolism. Eur Respir J 2015;45:1323-31.

32. Lankeit $M$, Jiménez D, Kostrubiec $M$, et al. Validation of N-terminal pro-brain natriuretic peptide cut-off values for risk stratification of pulmonary embolism. Eur Respir J 2014;43:1669-77.

33. Kabrhel C, Rosovsky R, Channick R, et al. A multidisciplinary pulmonary embolism response team: initial 30-month experience with a novel approach to delivery of care to patients with submassive and massive pulmonary embolism. Chest 2016;150:384-93.

34. Frémont B, Pacouret G, Jacobi D, Puglisi R, Charbonnier B, de Labriolle A. Prognostic value of echocardiographic right/left ventricular end-diastolic diameter ratio in patients with acute pulmonary embolism: results from a monocenter registry of 1,416 patients. Chest 2008;133:358-62.

35. Lobo JL, Holley A, Tapson V, the PROTECT and the RIETE investigators, et al. Prognostic significance of tricuspid annular displacement in normotensive patients with acute symptomatic pulmonary embolism. J Thromb Haemost 2014;12:1020-7.

36. Pruszczyk P, Goliszek S, Lichodziejewska B, et al. Prognostic value of echocardiography in normotensive patients with acute pulmonary embolism. JACC Cardiovasc Imaging 2014;7:553-60.

37. Tu T, Toma C, Tapson VF, et al. A prospective, single-arm, multicenter trial of catheter-directed mechanical thrombectomy for intermediate-risk acute pulmonary embolism: the FLARE study. JACC Cardiovasc Interv 2019;12:859-69.

38. Konstantinides SV, Meyer G. The 2019 ESC guidelines on the diagnosis and management of acute pulmonary embolism. Eur Heart J 2019;40:3453-5.

39. Kearon C, Akl EA, Ornelas J, et al. Antithrombotic therapy for VTE disease: CHEST guideline and expert panel report [published correction appears in. Chest. 2016 Oct;150(4):988]. Chest 2016;149:315-52. 